\title{
Crescimento, maturação, aptidão física, força explosiva e habilidades motoras específicas. Estudo em jovens futebolistas e não futebolistas do sexo masculino dos 12 aos 16 anos de idade
}

\author{
A. Seabra \\ J.A. Maia \\ R. Garganta \\ Faculdade de Ciências do Desporto e de Educação Física \\ Universidade do Porto, Porto, Portugal
}

https://doi.org/10.5628/rpcd.01.02.22

\section{RESUMO}

Este estudo investiga o impacto da maturação, da selecção e do treino na estrutura somática (ES), na aptidão física (AF), na força explosiva (FE) e nas habilidades motoras específicas (HME) de jovens jogadores de futebol. A amostra é constituída por 226 sujeitos com idades compreendidas entre os 12 e os 16 anos distribuídos por 3 grupos (G1-infantis; G2iniciados e G2-juvenis) de jogadores de futebol (JF) e de jovens sedentários (JS) do mesmo escalão etário. As medidas somáticas analisadas incluíram a altura, o peso, os perímetros, os diâmetros e pregas de adiposidade subcutânea que permitiram estimar dois compartimentos da massa corporal e o somatótipo. O desenvolvimento genital foi avaliado através das tabelas descritas por Tanner (1962). A AF foi avaliada pela bateria de testes da AAHPERD relacionada com a performance e descrita em Kirkendall et al. (1987). A avaliação da FE foi realizada através do protocolo descrito por Bosco et al. (1983). As HME foram avaliadas de acordo com a bateria de testes da Federação Portuguesa de Futebol (1986). Os procedimentos estatísticos incluíram a média aritmética, desvio-padrão, tteste de medidas independentes e a ANCOVA. No G1 a maturação, a selecção e o treino não tiveram um efeito significativo favorecendo os JF relativamente aos JS na estrutura somática e na força explosiva. Pelo contrário, constatou-se que o treino tinha um impacto nos futebolistas na grande maioria das componentes da $\mathrm{AF}$, à excepção da força inferior e resistência aeróbica. No G2 a maturação, a selecção e o treino não tiveram um efeito favorecendo os JF no peso, no mesomorfismo, no ectomorfismo, no SE e no SCM. Pelo contrário, nos futebolistas, o treino tinha um impacto, na massa gorda, no endomorfismo e na grande maioria das componentes da AF, exceptuando a força inferior e resistência aeróbica. No G3, e de igual forma, não houve um efeito marcado da maturação, da selecção e do treino favorecendo os JF relativamente aos JS no endomorfismo, no SE e no SCM. Contudo, constatou-se, através dos resultados apresentados pelos futebolistas, que o treino tinha um impacto no peso, na massa magra, e na grande maioria das componentes da AF, exceptuando a força inferior e a resistência aeróbica.

Palavras-chave: Crescimento; Maturação; Aptidão Física; Força Explosiva; Habilidades Motoras.

\begin{abstract}
Growth, maturation, physical fitness, explosive strength and specific motor skills. A study in Portuguese young soccer players and sedentary young people from 12 to 16 years old.
\end{abstract}

This study had the following purposes: to investigate the impact of selection and training on body build, physical fitness (PF), explosive strenght (ES) and specific motor skills (SMS) in Portuguese young soccer players.

Sample included 226 subjects, 12 through 16 years of age, divided in 3 groups (G1-infants; G2-beginners; G3-juveniles) of soccer players (SP) and sedentary subjects (SS) of the same age. Somatic measures included height, weight, girths, breadths and skinfolds. Body composition assessed fat mass and the lean mass with Boileau et al. (1985) formula. Somatotype was rated according to the Heath-Carter method (1967). Genital development was evaluated by the Tanner method (1962). Physical fitness was measured by the AAHPERD (1987) test battery performance related. The evaluation of the ES was accomplished through the Bosco (1983) procedures, and SMS with the test battery of the Federação Portuguesa de Futebol (1986). Statistical procedures included: mean, standard deviation, Qui-Square, the t-test and ANCOVA.

In G1 maturation, selection and training had no relevant effect favouring SP in comparison with SY in the somatic structure and ES. On the contrary, it was verified that training had an impact on SP in the great majority of components of the PF except standing long jump and 12 minute run.

In G2 maturation, selection and training had no effect favouring $\mathrm{SP}$, in the weight, meso and ecto somatotype components, SJ and $\mathrm{CMJ}$. On the other hand, on SP, training had an impact in fat mass, endomorphy and in the majority of the components of the $\mathrm{PF}$, but not standing long jump and 12 minute run. In G3 there was no remarkable effect of maturation, selection and training favouring SP relatively to SY, in the endomorphy, SJ and CMJ. On the contrary, it was stated, on SP, that training had an impact in weight, in lean mass and in the great majority components of PF but not standing long jump and 12 minute run.

Key Words: Growth, Maturation, Physical Fitness, Explosive strength, Motor skills. 


\section{INTRODUÇÃO}

A "evolução" do Futebol, enquanto jogo desportivo colectivo, tem passado, cada vez mais, pelo estudo e sistematização de elementos relativos a duas realidades interdependentes: o jogo e o jogador (25). No que se refere ao jogo, têm sido realizados estudos com diferentes focagens e alcances diversos (ver por exemplo: 1, 19, 23, 26, 28, 44). O mesmo tem ocorrido para o "indivíduo que joga", isto é, o jogador. Contudo, os trabalhos efectuados circunscrevem-se, essencialmente, ao Futebol de alto nível e ao jogador sénior.

No que concerne à criança e ao jovem jogador de Futebol importa salientar que as pesquisas são escassas. Tal facto não parece ser muito compreensível dado que o jovem atleta se encontra numa das primeiras etapas da sua preparação e formação e que visam o alto rendimento desportivo. Torna-se imperioso conhecer e sistematizar a maior quantidade e diversidade de informação acerca dos jovens que são submetidos, desde muito cedo, a actividades físicas organizadas, altamente especializadas e sistemáticas, sobretudo no que à resposta ao treino e competição dizem respeito. Facilmente podemos constatar que os jovens, futebolistas e não futebolistas, da mesma idade e sexo, apresentam diferenças significativas ao nível dos aspectos somáticos, da aptidão física geral e específica, e das respectivas habilidades. Contudo, nem sempre é claro se tal diferenciação é devida ao treino, à variabilidade maturacional que caracteriza os jovens no período pubertário, ou ao processo de selecção de jovens utilizado no Futebol. Parece-nos fundamental esclarecer de modo adequado estas questões.

O treino e a actividade física regular são geralmente interpretados como tendo uma influência favorável no crescimento, na maturação e na aptidão física da criança e do jovem (37). No entanto, a performance motora dos adolescentes do sexo masculino está significativamente relacionada com o seu estatuto maturacional. Os rapazes maturacionalmente avançados evidenciam, geralmente, melhores performances do que os atrasados na maturação (36). Segundo Bailey \& Mirwald (6), a variabilidade do estatuto maturacional caracteriza os jovens que praticam desporto, sendo especialmente evidente no período pubertário. De facto, nos rapazes, entre os 9 e os 16 anos, as variações associadas com a maturação biológica são muito significativas (32). Assim, ao afirmar-se que os jovens atletas são diferentes dos não atletas da sua idade e sexo, devemos questionar se tal é devido ao treino ou à variabilidade do processo de maturação, dado que uma grande parte das diferenças nas dimensões, forma, composição do corpo e performance é governada pelo estatuto maturacional (34). Contudo, além do treino e variação no estatuto maturacional, outro factor parece perfilar-se determinante, isto é, o processo de selecção para um determinado desporto. Os jovens atletas onde se vislumbra algum sucesso na sua resposta ao treino e competição, são um grupo altamente seleccionado, tomando por base geralmente as suas habilidades, e em alguns desportos, o tamanho dos sujeitos $(6,32)$. Consideramos, pois, relevante identificar os efeitos do treino intensivo e sistemático, da maturação e do processo de selecção, sobre os aspectos somáticos, aptidão física geral e específica e habilidades nos praticantes desta modalidade. Este motivo é tanto mais importante quando se sabe que nunca foi realizado nenhum estudo sobre esta temática em Portugal, e que são bem escassas as pesquisas publicadas internacionalmente.

O presente estudo tem, pois, por motivo central identificar o efeito da maturação, da selecção e do treino na estrutura somática, na aptidão física, na força explosiva e nas habilidades motoras específicas em jovens futebolistas.

\section{MATERIAL E MÉTODOS}

\subsection{Amostra}

A amostra é constituída por 226 sujeitos do sexo masculino de idade cronológica compreendida entre os 12 e os 16 anos. Cento e trinta e nove (139) são praticantes de Futebol federado, pertencentes a equipas de níveis competitivos diferenciados: 47 integrados numa das melhores equipas nacionais; 48 pertencem a uma equipa que "luta" pelo apuramento para as fases finais; e 44 a uma outra empenhada na subida aos Campeonatos Nacionais. Oitenta e sete são estudantes do $2^{\circ}$ e $3^{\circ}$ Ciclo do Ensino Básico, que nunca praticaram de modo regular qualquer actividade desportiva, excepção feita para as aulas 
curriculares de Educação Física.

Os sujeitos da amostra foram distribuídos por três escalões existentes na modalidade Futebol: infantis (com idade cronológica entre os 10 e os 12 anos; futebolistas $=11.74 \pm 0.44$; não futebolistas $=11.97 \pm 0.49$ ), iniciados (entre os $13 \mathrm{e}$ os 14 anos; futebolistas $=13.52 \pm 0.50$, não futebolistas $=13.53 \pm 0.50$ ) e juvenis (entre os $15 \mathrm{e}$ os 16 anos de idade; futebolistas $=16.09 \pm 0.54$, não futebolistas $=15.93 \pm 0.54$ ).

\subsection{Metodologia}

\subsubsection{Estrutura somática e maturaçãa}

Foram efectuadas 10 medidas somáticas, incluindo, além da altura e do peso, diâmetros, perímetros e pregas de adiposidade subcutânea. O protocolo utilizado foi o proposto pelo International Working Group on Kinanthropometry (IWGK), descrito por Ross \& Marfell-Jones (45) e por Borms (14). A composição corporal incluiu o cálculo da massa gorda e da massa isenta de gordura de acordo com as propostas de Boileau et al. (12). O somatótipo foi determinado de acordo com o método de Heath-Carter (29). O desenvolvimento genital foi avaliado através das cartas descritas por Tanner (50).

No Quadro 1 está referenciada a distribuição dos sujeitos dos dois grupos por nível de maturação sexual secundária.

Quadro 1 - Distribuição dos elementos da amostra pelos diferentes estádios de maturação, de acordo com o escalão.

\begin{tabular}{lcccccccccc} 
& \multicolumn{1}{c}{ Futebolistas } & \multicolumn{1}{c}{ Não Futebolistas } \\
\hline Estádio de & & & & & & & & & & \\
Maturação & 1 & 2 & 3 & 4 & 5 & 1 & 2 & 3 & 4 & 5 \\
Infantis & 5 & 32 & 9 & - & - & 7 & 20 & 2 & - & - \\
Iniciados & - & 7 & 17 & 20 & 3 & - & 13 & 6 & 9 & 2 \\
Juvenis & - & - & 16 & 30 & - & - & - & 2 & 17 & 9
\end{tabular}

\subsubsection{Aptidão Física}

A avaliação da aptidão física foi efectuada de acordo com a bateria de testes propostos pela AAHPERD relacionada com a performance e descrita em
Kirkendall et al. (31). Esta bateria propõe-se avaliar a aptidão física em função das seguintes componentes: (1) força superior - elevações na barra; (2) força média - abdominais; (3) força inferior - salto horizontal; (4) agilidade - corrida vai e vem; (5) velocidade - corrida de 50 metros; e (6) corrida de 12 minutos - resistência.

\subsubsection{Força Explosiva}

A avaliação da força explosiva foi realizada através do protocolo descrito por Bosco et al. (16). Para o efeito cada sujeito realizou três saltos: (1) salto vertical máximo a partir de uma posição estática (SE); (2) salto vertical máximo com contramovimento (SCM); e (3) saltos verticais máximos consecutivos durante um período de 15 segundos (PMM).

\subsubsection{Habilidades Motoras Específicas}

A avaliação das habilidades motoras específicas do Futebol foi efectuada com base numa bateria de testes da Federação Portuguesa de Futebol (21). Os testes pretendem descrever o nível de aptidão nas seguintes habilidades: (1) domínio e controlo da bola com o pé; (2) domínio e controlo da bola com a cabeça; (3) dribling / passe; (4) passe; (5) remate; (6) condução / velocidade.

\subsection{Procedimentos Estatísticos}

Os procedimentos estatísticos utilizados foram os seguintes: média aritmética, desvio-padrão, teste de Qui-quadrado, t-teste de medidas independentes e análise de covariância (ANCOVA). O programa estatístico utilizado foi o Systat 5.0 e o nível de significância foi mantido em $5 \%$.

\section{RESULTADOS}

Os resultados das variáveis serão apresentados (numa tabela e somatocarta) pelos diferentes escalões competitivos. 


\subsubsection{Infantis}

Quadro 2 - Média (x), desvio padrão (sd), valor da estatística t e significado estatístico (p) da diferença de médias entre os infantis com diferente prática desportiva.

\begin{tabular}{lcccc} 
Variáveis & $\begin{array}{c}\text { Futebolistas } \\
\mathbf{x} \pm \mathbf{s d}\end{array}$ & $\begin{array}{c}\text { Não Futebolistas } \\
\mathbf{x} \pm \mathbf{s d}\end{array}$ & $\mathrm{t}$ & $\mathrm{p}$ \\
\hline Medidas Somáticas & & & & \\
Altura & $149.14 \pm 6.74$ & $147.46 \pm 6.53$ & 1.06 & 0.295 \\
Peso & $42.53 \pm 5.62$ & $42.11 \pm 7.82$ & 0.27 & 0.787 \\
Composição Corporal & & & & \\
Massa gorda & $6.44 \pm 2.82$ & $7.76 \pm 4.72$ & -1.51 & 0.135 \\
Massa magra & $36.09 \pm 3.84$ & $34.31 \pm 3.81$ & 1.96 & 0.053 \\
Somatótipo & & & & \\
Endomorfismo & $2.50 \pm 1.10$ & $3.14 \pm 1.86$ & -1.87 & 0.064 \\
Mesomorfismo & $4.44 \pm 0.58$ & $4.58 \pm 0.84$ & -0.87 & 0.385 \\
Ectomorfismo & $2.77 \pm 0.93$ & $2.60 \pm 1.13$ & 0.69 & 0.488 \\
Aptidão Física & & & & \\
Elevaçães na barra & $0.94 \pm 0.98$ & $0.79 \pm 1.13$ & 0.60 & 0.551 \\
Abdominais & $38.91 \pm 7.68$ & $28.25 \pm 7.25$ & 5.92 & 0.000 \\
Salto horizontal & $1.69 \pm 0.18$ & $1.58 \pm 0.17$ & 2.65 & 0.01 \\
Corrida vai e vem & $10.69 \pm 1.61$ & $12.14 \pm 0.74$ & -4.48 & 0.000 \\
Corrida de 50 metros & $8.88 \pm 0.62$ & $9.74 \pm 0.74$ & -5.34 & 0.000 \\
Corrida de 12 minutos & $2346.09 \pm 348.86$ & $2035.71 \pm 486.03$ & 3.19 & 0.002 \\
Força Explosiva & & & & \\
SE & $26.30 \pm 4.50$ & $25.29 \pm 4.41$ & 0.94 & 0.350 \\
SCM & $26.57 \pm 4.89$ & $25.62 \pm 4.13$ & 0.86 & 0.391 \\
PMM & $26.04 \pm 5.99$ & $24.73 \pm 4.96$ & 0.97 & 0.334 \\
Hab. Mot. Específicas & & & & \\
Domínio e controlo pé & $30.80 \pm 26.2$ & $8.52 \pm 6.27$ & 4.49 & 0.000 \\
Domínio e controlo cabeça & $6.70 \pm 3.91$ & $3.69 \pm 2.10$ & 3.81 & 0.000 \\
Dribling / passe & $7.74 \pm 0.77$ & $12.11 \pm 3.46$ & -8.27 & 0.000 \\
Passe & $2.37 \pm 1.16$ & $1.59 \pm 1.02$ & 2.98 & 0.000 \\
Remate & $6.96 \pm 3.04$ & $5.00 \pm 3.17$ & 2.67 & 0.009 \\
Condução / velocidade & $13.79 \pm 0.82$ & $17.22 \pm 2.95$ & -7.48 & 0.000
\end{tabular}

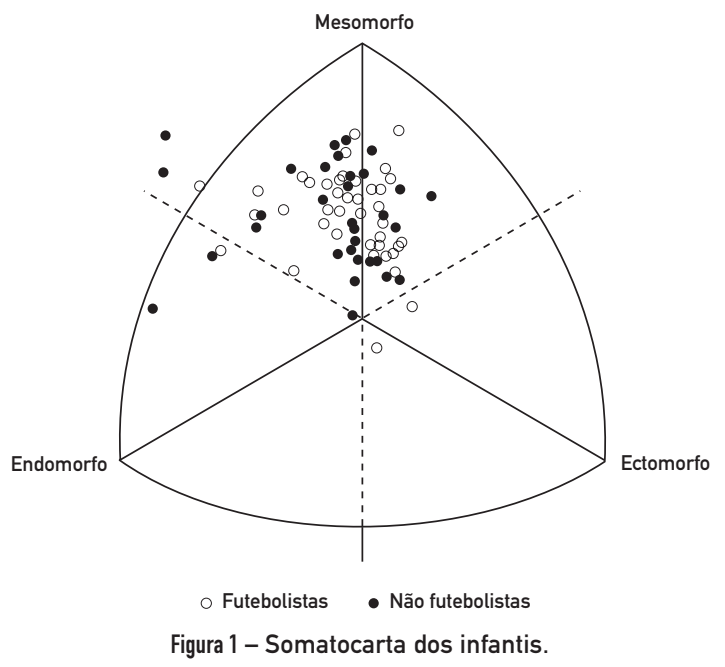

No escalão de infantis, os resultados encontrados revelaram diferenças estatisticamente significativas favorecendo os futebolistas, na maturação genital, nos abdominais, no salto horizontal, na corrida vai e vem, na corrida de 50 metros, na corrida de 12 minutos e em todas as habilidades motoras específicas.

Ao removermos o efeito da maturação através da ANCOVA, constatamos que as diferenças existentes continuaram a ser estatisticamente significativas, à excepção do salto horizontal e da corrida de 12 minutos. 
3.1.2. Iniciados

Quadro 3 - Média (x), desvio padrão (sd), valor da estatística t e significado estatístico ( $p$ ) da diferença de médias entre os iniciados com diferente prática desportiva.

\begin{tabular}{lcccc} 
Variáveis & $\begin{array}{c}\text { Futebolistas } \\
\mathbf{x} \pm \mathbf{s d}\end{array}$ & $\begin{array}{c}\text { Não Futebolistas } \\
\mathbf{x} \pm \mathbf{s d}\end{array}$ & $\mathbf{t}$ & $\mathbf{p}$ \\
\hline Medidas Somáticas & & & & \\
Altura & $162.41 \pm 8.27$ & $155.48 \pm 9.84$ & 3.36 & 0.001 \\
Peso & $52.31 \pm 8.21$ & $48.86 \pm 11.70$ & 1.53 & 0.129 \\
Composição Corporal & & & & \\
Massa gorda & $6.79 \pm 1.95$ & $8.73 \pm 5.38$ & -2.26 & 0.026 \\
Massa magra & $45.52 \pm 7.04$ & $40.39 \pm 7.62$ & 3.02 & 0.003 \\
Somatótipo & & & & \\
Endomorfismo & $1.95 \pm 0.50$ & $3.07 \pm 1.83$ & -3.98 & 0.000 \\
Mesomorfismo & $4.15 \pm 0.99$ & $4.55 \pm 1.50$ & -1.43 & 0.156 \\
Ectomorfismo & $3.30 \pm 0.81$ & $2.96 \pm 1.10$ & 1.55 & 0.124 \\
Aptidão Física & & & & \\
Elevações na barra & $3.34 \pm 2.57$ & $0.87 \pm 1.20$ & 4.99 & 0.000 \\
Abdominais & $43.91 \pm 5.87$ & $32.90 \pm 7.36$ & 7.33 & 0.000 \\
Salto horizontal & $1.89 \pm 0.20$ & $1.70 \pm 0.28$ & 3.41 & 0.001 \\
Corrida vai e vem & $10.76 \pm 0.83$ & $11.66 \pm 0.98$ & -4.38 & 0.000 \\
Corrida de 50 metros & $8.42 \pm 0.40$ & $9.36 \pm 1.17$ & -5.10 & 0.000 \\
Corrida de 12 minutos & $2553.11 \pm 255.75$ & $2245.16 \pm 507.27$ & 3.54 & 0.001 \\
Força Explosiva & & & & \\
SE & $30.30 \pm 4.78$ & $28.04 \pm 6.31$ & 1.81 & 0.075 \\
SCM & $31.39 \pm 4.89$ & $28.77 \pm 6.94$ & 1.96 & 0.053 \\
PMM & $31.98 \pm 5.53$ & $26.50 \pm 6.63$ & 3.96 & 0.000 \\
Hab. Mot. Específicas & & & & \\
Domínio e controlo pé & $47.66 \pm 45.12$ & $12.73 \pm 11.39$ & 4.15 & 0.000 \\
Domínio e controlo cabeça & $9.55 \pm 7.75$ & $4.90 \pm 4.71$ & 2.95 & 0.004 \\
Dribling / passe & $8.23 \pm 1.13$ & $11.68 \pm 3.23$ & -6.73 & 0.000 \\
Passe & $2.79 \pm 1.6$ & $2.10 \pm 1.54$ & 1.86 & 0.06 \\
Remate & $6.51 \pm 3.35$ & $4.77 \pm 3.03$ & 2.31 & 0.02 \\
Condução / velocidade & $14.04 \pm 1.48$ & $16.51 \pm 2.57$ & -5.37 & 0.000
\end{tabular}

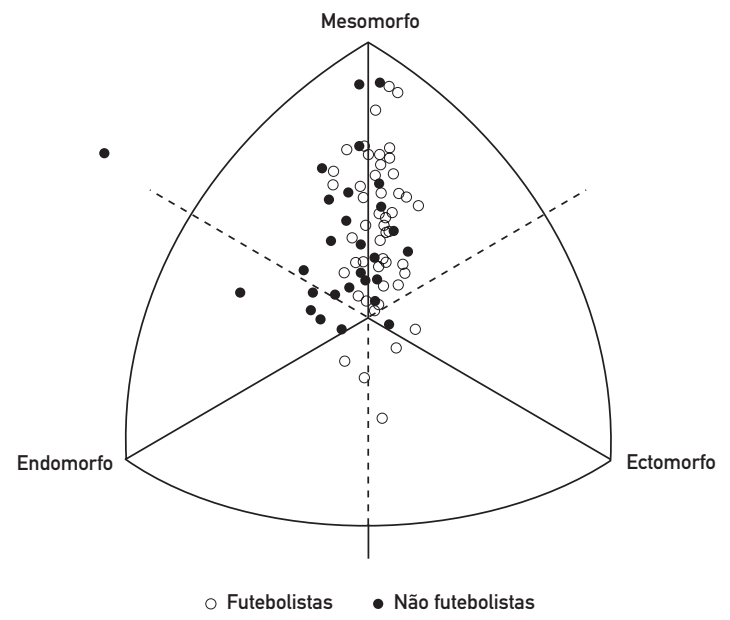

Figura 2 - Somatocarta dos iniciados.
No escalão de iniciados existem diferenças estatisticamente significativas favorecendo os futebolistas na altura, na massa gorda, na massa magra, no endomorfismo, no estatuto maturacional, nas elevações na barra, nos abdominais, no salto horizontal, na corrida vai e vem, na corrida de 50 metros, na corrida de 12 minutos, na PMM e em todas as habilidades à excepção da prova de passe. Contudo, ao removermos o efeito da maturação através da ANCOVA, constatamos que deixaram de se verificar diferenças estatisticamente significativas na altura, na massa magra, no salto horizontal, na corrida de 12 minutos e na PMM. 
3.1.3. Juvenis

Quadro 4 - Média (x), desvio padrão (sd), valor da estatística t e significado estatístico (p) da diferença de médias entre os juvenis com diferente prática desportiva.

\begin{tabular}{|c|c|c|c|c|}
\hline Variáveis & $\begin{array}{l}\text { Futebolistas } \\
\qquad \mathrm{x} \pm \mathrm{sd}\end{array}$ & $\begin{array}{l}\text { Não Futebolistas } \\
\qquad \pm \pm \text { sd }\end{array}$ & $\mathrm{t}$ & $\mathrm{p}$ \\
\hline \multicolumn{5}{|l|}{ Medidas Somáticas } \\
\hline Altura & $173.41 \pm 6.66$ & $167.99 \pm 7.76$ & 3.19 & 0.002 \\
\hline Peso & $70.38 \pm 6.41$ & $58.95 \pm 8.85$ & 6.43 & 0.000 \\
\hline \multicolumn{5}{|l|}{ Composição Corporal } \\
\hline Massa gorda & $10.85 \pm 3.17$ & $8.93 \pm 4.92$ & 2.05 & 0.044 \\
\hline Massa magra & $59.53 \pm 5.15$ & $50.02 \pm 6.89$ & 6.77 & 0.000 \\
\hline \multicolumn{5}{|l|}{ Somatótipo } \\
\hline Endomorfismo & $2.39 \pm 0.76$ & $2.51 \pm 1.55$ & -0.46 & 0.644 \\
\hline Mesomorfismo & $4.59 \pm 1.08$ & $4.06 \pm 0.94$ & 2.11 & 0.038 \\
\hline Ectomorfismo & $2.21 \pm 1.03$ & $3.14 \pm 1.39$ & -3.29 & 0.002 \\
\hline \multicolumn{5}{|l|}{ Aptidão Física } \\
\hline Elevações na barra & $5.13 \pm 3.61$ & $2.64 \pm 1.97$ & 3.35 & 0.001 \\
\hline Abdominais & $50.26 \pm 6.30$ & $34.75 \pm 6.87$ & 9.93 & 0.000 \\
\hline Salto horizontal & $2.15 \pm 0.14$ & $1.96 \pm 1.26$ & 4.01 & 0.000 \\
\hline Corrida vai e vem & $10.18 \pm 0.65$ & $10.87 \pm 0.83$ & -3.99 & 0.000 \\
\hline Corrida de 50 metros & $7.36 \pm 0.35$ & $8.41 \pm 0.64$ & -9.18 & 0.000 \\
\hline Corrida de 12 minutos & $2769.65 \pm 335.82$ & $2502.68 \pm 420.13$ & 3.01 & 0.004 \\
\hline \multicolumn{5}{|l|}{ Força Explosiva } \\
\hline $\mathrm{SE}$ & $34.52 \pm 5.09$ & $32.26 \pm 5.17$ & 1.84 & 0.069 \\
\hline SCM & $35.89 \pm 5.17$ & $33.81 \pm 5.47$ & 1.64 & 0.105 \\
\hline PMM & $34.26 \pm 6.96$ & $29.21 \pm 9.81$ & 2.59 & 0.012 \\
\hline \multicolumn{5}{|l|}{ Hab. Mot. Específicas } \\
\hline Domínio e controlo pé & $55.69 \pm 34.19$ & $17.71 \pm 19.6$ & 5.36 & 0.000 \\
\hline Domínio e controlo cabeça & $14.69 \pm 9.13$ & $6.71 \pm 7.04$ & 3.96 & 0.000 \\
\hline Dribling / passe & $8.83 \pm 1.28$ & $10.41 \pm 2.98$ & -3.16 & 0.002 \\
\hline Passe & $2.67 \pm 1.41$ & $2.00 \pm 1.41$ & 1.98 & 0.04 \\
\hline Remate & $6.04 \pm 3.13$ & $4.96 \pm 2.32$ & 1.58 & 0.11 \\
\hline Condução / velocidade & $13.76 \pm 0.86$ & $15.33 \pm 2.31$ & -4.2 & 0.000 \\
\hline
\end{tabular}

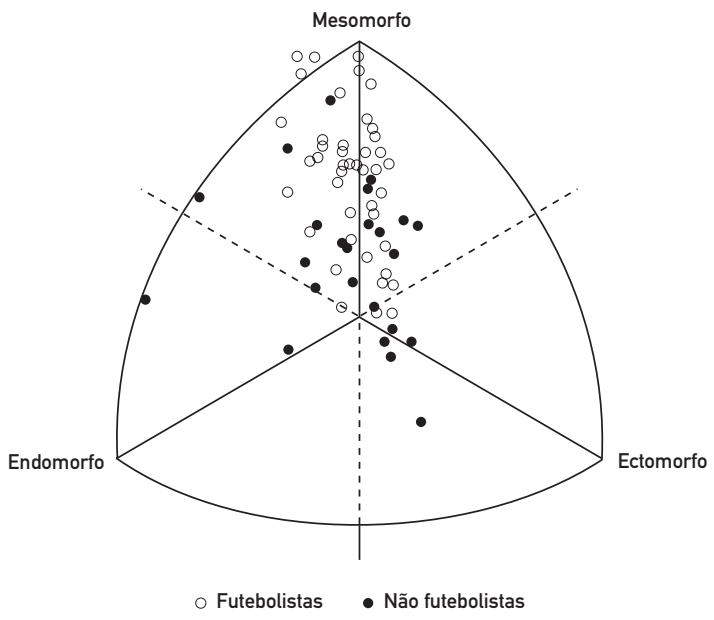

Figura 3 - Somatocarta dos juvenis.
No escalão de juvenis, os futebolistas apresentam resultados significativamente superiores na altura, no peso, na massa gorda, na massa magra, no mesomorfismo, no ectomorfismo, no estatuto maturacional, nas elevações na barra, nos abdominais, no salto horizontal, na corrida vai e vem, na corrida de 50 metros, na corrida de 12 minutos, na PMM e em todas as habilidades à excepção da prova de remate.

No entanto, ao removermos o efeito da maturação através da ANCOVA, podemos constatar que à excepção do peso e da massa magra, as diferenças existentes deixaram de ser estatisticamente significativas na altura, na massa gorda, no mesomorfismo, no ectomorfismo, no salto horizontal, na corrida de 12 minutos e na PMM. 


\section{DISCUSSÃO}

\subsection{Estrutura somática e maturação}

\subsubsection{Altura e peso}

No presente estudo, os futebolistas constituintes da nossa amostra manifestaram claramente, em ambos os escalões, resultados superiores aos não futebolistas nas medidas somáticas altura e peso. No que diz respeito à altura, foram encontradas diferenças estatisticamente significativas entre os futebolistas e os não futebolistas, no escalão de iniciados e de juvenis. Relativamente ao peso, as diferenças evidenciadas entre os dois grupos só se registaram significativas no escalão de juvenis. Por outro lado, constatou-se ainda, em todos os escalões, que os futebolistas apresentavam um estatuto maturacional mais avançado que os não futebolistas.

Estes resultados estão em concordância com os de outros autores $(6,32,35,37)$ que referem que grande parte dos rapazes atletas tendem a estar mais avançados em termos maturacionais do que os jovens da mesma idade cronológica que não praticam qualquer desporto. Este facto poderá explicar as diferenças dimensionais e ponderais dos jovens avançados maturacionalmente relativamente aos jovens atrasados, que são sempre mais baixos e leves. Daqui que se questione se tais diferenças entre jovens futebolistas e não futebolistas são devidas ao treino, à selecção ou, pelo contrário, resultantes exclusivamente do processo normal de crescimento somático e maturação.

A remoção do factor maturação mostrou a inexistência de diferenças significativas na altura. Este facto mostrou claramente que a altura é uma medida somática fortemente dependente da variância maturacional, a que se associa uma heritabilidade elevada, sobretudo nos parâmetros do salto pubertário.

Quando se considera o estatuto maturacional dos sujeitos, o treino regular não apresenta um efeito estimulador no crescimento estatural para além do que se espera do genótipo dos sujeitos $(6,32,38)$ em condições adequadas do envolvimento. No peso, mesmo após a remoção do efeito da maturação, as diferenças mantiveram-se estatisticamente significativas. Tal sugere que o treino é um dos agentes que influencia esta medida somática. Malina (37) refere que rapazes adolescentes tendem a aumentar o seu peso em associação à actividade físico-desportiva regular. Estes ganhos devem estar relacionados com o aumento da massa isenta de gordura (massa muscular) que ocorre durante e após o pico de velocidade de altura e se prolonga até ao adulto jovem. A este efeito há a acrescentar os estímulos provenientes do próprio treino sobre a massa isenta de gordura.

\subsubsection{Composição corporal}

Os resultados encontrados neste trabalho mostraram no escalão de infantis e de iniciados, valores superiores de massa gorda dos não futebolistas relativamente aos futebolistas. No escalão de juvenis os futebolistas apresentaram resultados superiores, contudo as diferenças só se revelaram estatisticamente significativas no escalão de iniciados e de juvenis.

Do mesmo modo, na massa isenta de gordura, só se registaram diferenças estatisticamente significativas entre os grupos nos escalões de iniciados e de juvenis. Estes resultados estão em concordância com os de Bailey \& Martin (5) e de Malina (35) - os rapazes mais activos e os atletas infanto-juvenis possuem uma menor massa gorda e maior massa isenta de gordura (massa muscular) quando comparados com os moderadamente ou normalmente activos e os não atletas.

Contudo, e segundo alguns autores $(7,12)$, a composição corporal altera-se consideravelmente durante o processo normal de crescimento somático, sobretudo na fase pubertária e pós-pubertária sendo, por isso, extremamente difícil separar as alterações que são devidas ao treino daquelas que estão associadas ao crescimento e maturação. Após remoção do efeito maturacional verificou-se que na massa gorda as diferenças se mantinham estatisticamente significativas no escalão de iniciados. O mesmo não aconteceu no de juvenis. Na massa isenta de gordura as diferenças deixaram de ser estatisticamente significativas no escalão de iniciados e mantiveram-se no de juvenis. Beunen (8) e Malina (37) referem que nos rapazes a massa isenta de gordura (i. e. a massa muscular) está associada ao processo maturacional sendo que 
tal associação é relativamente fraca durante a infância e moderadamente forte no período pubertário. Os resultados encontrados salientam o efeito positivo da actividade física regular e o do treino no aumento da massa isenta de gordura particularmente no período pós-pubertário (escalão de juvenis).

\subsubsection{Somatótipo}

No que diz respeito à morfologia externa do corpo (somatótipo) e particularmente às suas componentes, pudemos constatar a existência de diferenças entre futebolistas e não futebolistas nos diferentes escalões.

No escalão de infantis, os somatótipos médios não diferiram significativamente entre os dois grupos. No escalão de iniciados, os não futebolistas apresentaram valores superiores na componente endomórfica. Após remoção do efeito da maturação as diferenças permaneceram estatisticamente significativas. Nos juvenis, os futebolistas revelaram resultados significativamente superiores na componente endomórfica e inferiores na ectomórfica. Contudo, após remoção do efeito da maturação as diferenças deixaram de ser estatisticamente significativas.

Neste estudo, pudemos constatar, tanto nos futebolistas como nos não futebolistas, que a componente mesomórfica é dominante. Esta componente diminui os seus resultados do escalão de infantis para o de iniciados para voltar a evidenciar valores mais elevados nos juvenis. Carter (18) refere que, nos rapazes atletas, a tendência para aumentar em mesomorfia é um fenómeno evidente da adolescência até à idade adulta. Este facto é igualmente verdadeiro para as populações não atléticas. Contudo, e de acordo com alguns autores $(20,30,49)$, a mesomorfia diminui ligeiramente nos rapazes, quer atletas quer não atletas, do take-off para o pico de velocidade da altura (PVA). Esta diminuição está provavelmente relacionada com o aumento em ectomorfia. Isto é, verifica-se primeiro um aumento da linearidade, para após o PVA se constatar um aumento do peso e uma predominância da mesomorfia. Nesta pesquisa os não futebolistas, em todos os escalões, revelam valores superiores na componente endomórfica. Tal circunstância é clara na literatura somatotipológica.

Carter (18) refere estudos de somatótipos realizados em atletas de alto nível mostrando a predominância da mesomorfia relativamente à endomorfia. Este padrão é diverso daquele presente em amostras de referência e em atletas de baixo nível competitivo. De acordo com este autor, o treino e a participação em desportos tendem a aumentar a mesomorfia e a diminuir a endomorfia.

\subsection{Aptidão física}

\subsubsection{Força superior}

Nesta componente da aptidão física, os futebolistas evidenciaram resultados superiores aos não futebolistas em todos os escalões.

No escalão de infantis, os valores apresentados pelos futebolistas apesar de superiores não foram significativamente diferentes dos manifestados pelos não futebolistas, uma vez que foram poucos os elementos da amostra que conseguiram realizar uma elevação na barra.

Nos escalões de iniciados e de juvenis, os resultados encontrados para os futebolistas foram muito superiores e significativamente diferentes dos apresentados pelos não futebolistas.

Na prova de elevações na barra, o efeito gravítico tem uma importância fundamental nas performances eventualmente realizadas, uma vez que o sujeito tem necessidade de mover e suportar o seu peso corporal contra a acção da gravidade. Fleishman (22) encontrou nesta prova uma correlação negativa entre o peso corporal e a performance, ou seja, quanto maior o peso corporal do sujeito piores são as performances realizadas. Esta relação é corroborada pelo facto do aumento do peso corporal e das dimensões lineares condicionarem alometricamente a performance (ver por exemplo 2). Enquanto a área de secção transversa dos músculos aumenta para $\mathrm{L}^{2}$, a massa é proporcional a $\mathrm{L}^{3}$, implicando uma desvantagem nítida dos sujeitos dimensionalmente superiores (ver 3). Safrit (46) adiciona a circunstância da performance nesta prova estar negativamente correlacionada com a gordura corporal. Neste estudo, os não futebolistas apresentam valores de composição corporal e de morfologia externa muito diferentes dos evidenciados pelos futebolistas. 
Relativamente à composição corporal, podemos verificar que no escalão de iniciados, os não futebolistas manifestam valores superiores de massa gorda $(+1.94 \mathrm{~kg})$ e menores de massa isenta de gordura $(-5.13 \mathrm{~kg})$.

No escalão de juvenis, os valores de massa gorda tornam-se semelhantes, enquanto que na massa isenta de gordura as diferenças entre futebolistas e não futebolistas se tornam muito superiores $(9.51 \mathrm{~kg})$. Por outro lado, nestes dois escalões, os futebolistas revelam uma morfologia externa diferenciada da dos não futebolistas. Enquanto nos futebolistas predomina a componente mesomórfica que exprime a robustez do esqueleto e o desenvolvimento da musculatura, nos não futebolistas prevalece a componente endomórfica que, como se sabe, exprime a tendência para a adiposidade. Estes factos justificam, também, parte do diferencial de resultados obtidos.

No entanto, e como suspeitávamos que a maturação fosse um factor capaz de influenciar estes resultados, removemos o seu efeito. Após a remoção, constatámos que as diferenças existentes entre futebolistas e não futebolistas se mantinham estatisticamente significativas. Estes resultados sugerem que o treino é um dos factores responsáveis pela melhoria da performance desta componente da aptidão física.

\subsubsection{Força média}

Os futebolistas apresentaram valores significativamente superiores aos evidenciados pelos não futebolistas em todos os escalões. Resultados diferentes foram encontrados por Verschuur (51) e Beunen et al. (11) em amostras de jovens activos e inactivos dado não encontrarem diferenças de realce entre eles. No entanto, Beunen (8) refere que nos rapazes com idades compreendidas entre os 12 e os 13 anos este teste está negativamente correlacionado com a idade esquelética, contrariamente ao que acontece acima dos 14 anos de idade onde todas as capacidades motoras estão positivamente correlacionadas com a idade esquelética. Desse modo, atendendo ao citado anteriormente e ao facto de sabermos que todas as componentes da aptidão física revelam alterações em função do crescimento e da maturação, procurámos conhecer o possível efeito que a maturação poderia ter tido sobre os resultados encontrados.

Nesta prova, mesmo após a remoção do efeito da maturação as diferenças existentes entre futebolistas e não futebolistas mantêm-se significativas. Estes resultados vêm assim mostrar, uma vez mais, que o treino tem uma influência relevante na melhoria da capacidade de força-resistência da musculatura abdominal.

\subsubsection{Força inferior}

Os resultados encontrados nesta componente evidenciam valores superiores nos futebolistas em todos os escalões. As diferenças encontradas foram sempre estatisticamente significativas.

Resultados diferentes foram encontrados por Verschuur (51) no estudo longitudinal que realizou com crianças activas e inactivas dos 13 aos 16 anos. Nesse trabalho, e na prova de salto horizontal, não foram encontradas diferenças entre as crianças activas e inactivas.

Tal como referimos anteriormente, no sexo masculino a força muscular aumenta linearmente com a idade cronológica desde o inicío da infância até aproximadamente os 13/14 anos, havendo a partir daí uma marcada aceleração no seu desenvolvimento $(38,24)$.

É pois natural que, em cada intervalo de idade, os rapazes avançados maturacionalmente apresentem uma maior força muscular do que os rapazes atrasados na maturação. Essas diferenças na força entre grupos de diferente maturação são mais evidentes entre os 13 e os 16 anos de idade (24). Ao removermos o efeito da maturação verificamos, em todos os escalões, que as diferenças deixaram de ser significativas. Isto sugere a elevada importância do factor maturacional e a eventual ausência do efeito substancial do treino.

\subsubsection{Agilidade}

Nesta prova e em todos os escalões, os futebolistas evidenciaram valores significativamente superiores aos não futebolistas.

Verschuur (51) salientou as diferenças significativas de crianças e jovens activas relativamente aos inactivos, a que se parece associar um efeito interactivo maturação x treino. 
No Futebol, o único trabalho encontrado foi realizado por Garganta et al. (27) com jovens futebolistas de nível competitivo diferenciado (elite/ não elite). Na prova de agilidade, os futebolistas de elite evidenciaram valores significativamente inferiores relativamente aos de não elite. Pelo contrário, na pesquisa longitudinal realizada por Beunen et al. (11) com crianças activas e inactivas dos 13 aos 18 anos não foram encontradas diferenças de realce.

Deste modo, e a exemplo do fizemos com as outras componentes, procuramos saber se nesta prova as diferenças existentes entre futebolistas e não futebolistas eram devidas ao efeito da maturação. Após remoção da maturação as diferenças mantinham-se estatisticamente significativas, o que nos leva a pensar que o treino tem uma influência positiva no aumento da performance da corrida de vai e vem.

\subsubsection{Velocidade}

Os valores médios obtidos para a corrida de 50 metros evidenciaram em todos os escalões uma "superioridade" estatisticamente significativa dos futebolistas relativamente aos não futebolistas. Sobral (48) refere que do ponto de vista "evolutivo" se verifica um incremento acentuado da velocidade de deslocamento, avaliada através de provas de corrida curta (20 a 50 metros), dos 5 aos 16 anos de idade. $\mathrm{O}$ mesmo autor refere ainda que nos rapazes entre os 10 e os 13 anos se observa um aumento regular das prestações. Marques et al. (40) salientam este aspecto ao referir que nos rapazes, a partir do período pubertário, há uma maior percentagem de massa muscular e um desenvolvimento superior da capacidade anaeróbica o que como sabemos determina os resultados desta componente. Desse modo, e uma vez que a componente velocidade é condicionada pelo desenvolvimento geral do indivíduo e muito particularmente pela sua maturação biológica (39), torna-se importante saber se os resultados encontrados nesta prova eram devidos à maturação. Para o efeito removemos a maturação tendo constatado que as diferenças até aí existentes se mantinham estatisticamente significativas. Estes resultados parecem evidenciar que a maturação não tem uma influência significativa sobre esta componente da aptidão física. Pelo contrário, o treino e a selecção utilizada no Futebol poderão ser os principais factores responsáveis pelos resultados obtidos.

\subsubsection{Resistência aeróbica}

Os futebolistas percorreram distâncias muito superiores aos não futebolistas.

Segundo Mirwald et al. (41) e Marques et al. (40), os valores médios, nas provas de resistência aeróbica avaliadas indirectamente aumentam com a idade. Com o aparecimento da puberdade, os rapazes mostram um aumento da potência aeróbica que está claramente relacionado com o pico de velocidade da altura (41). Borms (13) confirma este aspecto ao referir que nos rapazes atletas, após os 12 anos de idade, há um aumento na sensibilidade ao treino do sistema cárdio-circulatório, decorrendo daqui um aumento da capacidade aeróbica. Durante a fase de crescimento pubertário regista-se um aumento da potência máxima aeróbica independentemente da prática ou não de actividade física. Contudo, a idade biológica parece influenciar a potência aeróbica, dado existir uma correlação positiva entre esses dois factores. Bailey (4) salienta que entre os 8 e os 15 anos de idade, os indivíduos maturacionalmente avançados revelam um maior consumo máximo de oxigénio do que os atrasados na maturação.

Vrijens \& Van Cauter (52) realizaram um estudo com jovens futebolistas com idades compreendidas entre os 10 e os 13 anos com o objectivo de avaliar a influência da idade biológica em diferentes componentes da aptidão física. Uma das componentes avaliadas foi a capacidade aeróbica numa prova de corrida de 6 minutos. Os resultados encontrados mostraram valores mais baixos de capacidade aeróbica nos indivíduos atrasados na maturação. Contudo, as diferenças encontradas não foram estatisticamente significativas, dada a redução da dimensão da amostra $(n=66)$.

Também aqui procurámos verificar se as diferenças existentes entre futebolistas e não futebolistas eram devidas ao efeito maturacional. Ao remover a maturação verificou-se que as diferenças deixaram de existir. Daqui que a maturação deva ser considerado o factor influenciador das diferenças de resultados desta prova. O treino por si só não parece ter um efeito positivo na melhoria da performance 
desta componente da aptidão física nos futebolistas estudados.

Beunen (8) reforça este aspecto ao referir as elevadas correlações entre estatuto maturacional e consumo máximo de oxigénio. Contudo, quando o consumo máximo foi expresso em termos relativos as correlações passaram a ser baixas e deixaram de ser significativas.

\subsection{Força explosiva}

No SE e no SCM, os resultados obtidos nos diferentes escalões não apresentam diferenças estatisticamente significativas. Contudo, os valores mais elevados expressos pelos futebolistas no teste do SE e no SCM poderão ser devidos a um melhor aproveitamento da energia elástica e da capacidade contráctil do músculo. Bosco et al. (17) referem que o potencial elástico do músculo esquelético humano é uma propriedade que pode ser melhorada através do treino. Deste modo, o aumento do número de anos de treino conduz a uma melhoria do aproveitamento da contracção concêntrica no ciclo de estiramento/ encurtamento. A comparação dos valores obtidos no SCM com os do SE permite constatar que os valores encontrados no SCM são superiores, facto que vem "confirmar" que o pré-alongamento muscular aumenta a força produzida durante a contracção concêntrica imediata.

No teste da PMM os futebolistas apresentaram valores superiores aos não futebolistas em todos os escalões. No entanto, as diferenças estatisticamente significativas só ocorrem nos escalões de iniciados e de juvenis.

Contudo, constatámos que após remoção do efeito da maturação as diferenças até aí existentes nos escalões de iniciados e de juvenis deixaram de existir. A maturação, nestes escalões, revelou-se um factor influenciador dos resultados, contribuindo dessa forma para diferenciar futebolistas e não futebolistas.

Segundo Beunen \& Malina (9), o facto dos níveis de força serem superiores nos indivíduos mais adiantados no estatuto maturacional reflecte em parte um maior tamanho corporal e uma maior quantidade de massa isenta de gordura. De acordo com estes autores, os indivíduos mais avançados no processo de maturação estão mais predispostos para conseguirem resultados superiores em tarefas que façam apelo à capacidade de força ou de forçavelocidade (potência), como por exemplo os saltos, os lançamentos e os sprints.

Desse modo, nos rapazes, as correlações entre a força muscular, a prestação desportiva e os indicadores de maturação esquelética e sexual tendem a ser mais elevadas entre os 13 e os 16 anos de idade, sendo mais evidentes, nesta faixa etária, as diferenças na força muscular entre os mais e os menos adiantados no processo de maturação. Do mesmo modo, Vrijens \& Van Cauter (52) ao estudarem a influência da maturação esquelética no desenvolvimento somático e na prestação motora de 66 jovens futebolistas, com idades cronológicas compreendidas entre os 10 e os 13 anos, concluiram que a capacidade motora força muscular é influenciada pela maturação esquelética.

Os resultados deste trabalho estão em concordância com os alcançados por Bosco \& Luhtanen (15) no estudo que realizaram com futebolistas e não futebolistas dos escalões de iniciados (14 anos) e de juvenis (16 anos). Nessa pesquisa, independentemente do escalão, os futebolistas apresentaram sempre valores superiores aos não futebolistas nos vários testes de força explosiva realizados. No entanto, as diferenças encontradas entre futebolistas e não futebolistas, nos diferentes testes, apenas foram estatisticamente significativas no escalão de juvenis.

O estatuto maturacional é um factor que influencia o nível de desenvolvimento da força muscular, nomeadamente nas formas de manifestação de forçavelocidade e de força máxima, com especial incidência entre os 13 e os 16 anos de idade. Nesta conformidade e concluindo, não podemos referir claramente se estes resultados são devidos ao treino, visto que o processo de maturação biológica influencia e condiciona os resultados alcançados pelos futebolistas e pelos não futebolistas.

\subsection{Habilidades motoras específicas}

Os resultados médios obtidos no presente estudo evidenciam, em todas as habilidades motoras específicas do Futebol, uma superioridade dos futebolistas em relação aos não futebolistas. A comparação destes resultados permitiu ainda 
verificar a existência de diferenças com significado estatístico em todas as habilidades específicas, à excepção do passe no escalão de iniciados e do remate no escalão de juvenis.

Mas a que serão devidas estas diferenças tão significativas existentes entre futebolistas e não futebolistas nas várias habilidades? Será que o treino e a maturação tiveram alguma influência nos resultados alcançados?

Nesta temática e contrariamente ao que fizemos nas anteriores, não removemos o efeito da maturação pois segundo alguns autores $(47,52,53)$ a maturação não parece ter uma grande influência sobre as habilidades motoras específicas. Pelo contrário, o treino e o número de anos de prática de Futebol parecem ter uma acção positiva na melhoria destas habilidades.

Vrijens \& Van Cauter (52) não encontraram nenhuma correlação entre a maturação e as habilidades específicas do Futebol, ao passo que com o treino e o número de anos de prática desta modalidade os coeficientes de correlação foram altamente significativos. Zaichkowsky et al. (53) referem igualmente que a experiência (aprendizagem e treino) é um factor crítico na aquisição das habilidades específicas, pelo que as crianças treinadas têm melhores performances do que as não treinadas. Schmidt (47) salienta este mesmo aspecto ao mencionar a existência de muitos factores que aumentam a capacidade de performance das habilidades específicas, realçando a experiência e a prática. Segundo este autor, os atletas de alto rendimento apresentam elevados níveis de habilidades, devido essencialmente à quantidade de tempo e de prática que aplicam na sua preparação. Daqui que um alto nível de prática de elevada qualidade seja um factor crítico no desenvolvimento das habilidades motoras específicas.

\section{CONCLUSÕES}

No escalão de infantis a maturação teve um efeito significativo favorecendo os futebolistas na força inferior e na resistência aeróbica. O treino teve um impacto nos resultados dos futebolistas na força média, na agilidade e na velocidade. A selecção, a maturação e o treino não evidenciaram qualquer efeito significativo favorecendo os futebolistas na estrutura somática e na força explosiva.

No escalão de iniciados a maturação teve um efeito significativo favorecendo os futebolistas na altura, na massa isenta de gordura, na força inferior, na resistência aeróbica e na PMM. O treino mostrou um impacto relevante nos futebolistas na massa gorda, no endomorfismo, na força superior e média, na agilidade e na velocidade. Pelo contrário, a maturação, a selecção e o treino não tiveram qualquer efeito nos futebolistas, no que se refere ao peso, mesomorfismo, ectomorfismo, SE e SCM. No escalão de juvenis a maturação teve um efeito significativo favorecendo os futebolistas na altura, na massa gorda, no mesomorfismo, na força inferior, na resistência aeróbica e na PMM. O treino mostrou um impacto nos resultados apresentados pelos futebolistas no peso, na massa isenta de gordura, na força superior e média, na agilidade e na velocidade. Contudo, não houve um efeito marcado da maturação, da selecção e do treino favorecendo os futebolistas, no endomorfismo, no SE e no SCM.

\section{CORRESPONDÊNCIA}

\section{José Maia}

Faculdade de Ciências do Desporto

e de Educação Física

Universidade do Porto

R. Dr. Plácido Costa, 91

4200.450 Porto

Portugal

[jmaia@fcdef.up.pt] 


\section{REFERÊNCIAS BIBLIOGRÁFICAS}

1. Ali A (1988). A Statistical Analysis of Tactical Movement Patterns in Soccer. In Reilly T, Lees A, Davids K, Murphy W (eds.); Science and Football, Proceedings of the First World Congress of Science and Football. London: E \& F.N. Spon. London, 302-308

2. Asmussen E, Heebol-Nielsen K (1955). A dimensional analysis of physical performance and growth in boys. Journal Applied Physiology 7: 593-603

3. Astrand P, Rodahl K (1980). Tratado de fisiologia do exercício. Interamericana. Rio de Janeiro

4. Bailey D (1976). The Growing Child and the Need for Physical Activity. In Albinson J, Andrew G (eds.). Child in Sport and Physical Activity. International Series on Sport Science. Baltimore: University Park Press, 3: 81-93

5. Bailey D, Martin A (1988). The Growing Child and Sport: Physiological Considerations. In Small F, Magill R, Ash M (eds.). Children in Sport. Champaign: Human Kinetics Books, 103-117 6. Bailey D, Mirwald R (1988). The Effects of Training on the Growth and Development of the Child. In Malina R (ed.). Young Athletes - Biological, Psychological and Educational Perspectives. Champaign: Human Kinetics Books, 33-47 7. Bailey D, Malina R, Mirwald R (1986). Physical Activity and Growth of the Child. In Falkner F, Tanner J (eds.). Human Growth. New York: Plenum Press, 2: 147-170

8. Beunen G (1990). Biological Maturation and Physical Performance. In Duquet W, Day J (eds.). Kinanthropometry IV Proceedings of the International Congress on Youth, Leisure and Physical Activity. London: E \& F. N. SPON, 215-235 9. Beunen G, Malina R (1988). Growth and Physical Performance Relative to The Timing of the Adolescent Spurt. Exercise Sport Science Review 16: 503-540

10. Beunen G, Claessens A, Ostyn M, Renson R, Simons J, Van Gerven D (1983). Motor Performance as Related to Somatotype in Adolescent Boys. In Brinkhorst R, Kemper H, Saris W (eds.). International Series on Sport Sciences - Children and Exercise. XI vol.15. Champaign: Human Kinetics Publishers, Inc., 279-284

11. Beunen G, Malina R, Renson R, Simons J, Ostyn M, Lefevre J (1992). Physical Activity and Growth, Maturation and Performance - A Longitudinal Study. Medicine Science in Sports and Exercise 24 (5): 576-585

12. Boileau R, Lohman T, Slaughter M (1985). Exercise and Body Composition of Children and Youth. Scandinavian Journal Sports Science 7: 17-27

13. Borms J (1986). The Child and Exercise - An Overview. Journal of Sports Sciences 4: 1-18

14. Borms J (1987). Kinanthropometry - a Post Graduate Course. Instituto Superior de Educação Física - Universidade Técnica de Lisboa

15. Bosco C, Luhtanen P (1992). Fisiologia e Biomeccanica Applicata al Calcio. In: Scienze e Sport. Roma. Società Stampa Sportiva

16. Bosco C, Luhtanen P, Komi P (1983). A Simple Method for Measurement of Mechanical Power in Jumping. European Journal Applied Physiology 50: 273-282

17. Bosco C, Pittera C, Rusko H, Rahkila P, Luhtanen P, Ito A, Droghetti P, Ziglio P (1981). New Tests for the Measurement of Anaerobic Capacity in Jumping and Leg Extensor Muscle Elasticity. Volleyball, I.F.V.A. Official Magazine 1: 22-30 18. Carter L (1988). Somatotypes of Children in Sports. In Malina R (ed.). Young Athletes - Biological, Psychological and
Educational Perspectives. Champaign: Human Kinetics Books, 153-165

19. Castelo J (1994). Futebol - Modelo Técnico-Táctico do Jogo. Faculdade de Motricidade Humana - Universidade Técnica de Lisboa

20. Faróis I (1982). Results of Constitutional and Motor Examinations of Male Athletes. Glasnik Antropoloskog Drustva Jugoslavije 19: 35-51

21. Federação Portuguesa de Futebol (1986). Habilidades e Destrezas do Futebol - Os Skills do Futebol. Editora Federação Portuguesa de Futebol. Lisboa

22. Fleishman E (1964). The Structure and Measurement of Physical Fitness. Prentice-Hall, Inc. Englewood Cliffs. New Jersey 23. Franks I, McGarry T (1996). The Science of Match Analysis. In Reilly T (ed). Science and Soccer. London: E \& F.N. Spon, 363-375

24. Froberg K, Lammert O (1996). Development of Muscle Strength During Childhood. In Bar-Or O (ed.). Child and Adolescent Athlete. Blackwell Science. Oxford

25. Garganta J (1991). Estudo Descritivo e Comparativo da Força Veloz e Força Explosiva em Jovens Praticantes de Futebol no Intervalo Etário 14-17 anos. Dissertação Apresentada às Provas de Aptidão Pedagógica e de Capacidade Científica. Faculdade de Ciências do Desporto e de Educação Física Universidade do Porto

26. Garganta J (1997). Modelação Táctica do Jogo de Futebol. Estudo da Organização da Fase Ofensiva em Equipas de Alto Rendimento. Dissertação Apresentada às Provas de Doutoramento. Faculdade de Ciências de Desporto e de Educação Física - Universidade do Porto

27. Garganta J, Maia J, Silva R, Natal A (1993). A Comparative Study of Explosive Leg Strength in Elite and Non-Elite Young Soccer Players. In Reilly T, Clarys J, Stibbe A (eds.). Science and Football II. London: E \& F. N. Spon, 304-306

28. Gréhaigne J (1992). L'Organisation du Jeu en Football. Editions Actio. Joinville-le-Pont

29. Heath B, Carter J (1967). A Modified Somatotype Method. American Journal Physiology Anthropology 27: 57-74 30. Hebbelinck M, Borms J (1978). Korperliches Wachstum und Leistungs - Faehigkeit bei Schulkindern (Physical Development and Performance Capacity in Schoolchildren). Leipzig: Barth 31. Kirkendall D, Gruber J, Johnson R (1987). Measurement and Evaluation for Physical Educators. Champaign: Human Kinetics Publishers, Inc

32. Malina R (1980). Physical Activity, Growth, and Functional Capacity. In Johnston F, Roche A, Susanne C (eds.). Human Physical Growth and Maturation. New York: Plenum Press, 303-327 33. Malina R (1988). Growth and Maturation of Young Athletes: Biological and Social Considerations. In Smoll F, Magill R, Ash M (eds.). Children in Sport. Champaign: Human Kinetics Books, 83-101

34. Malina R (1988). Biological Maturity Status of Young Athletes. In Malina R (ed). Young Athletes - Biological, Psychological and Educational Perspectives. Champaign: Human Kinetics Books, 121-140

35. Malina R (1988). Training for Sport and Puberty. In Laron Z, Rogol A (eds). Hormones and Sport. New York: Raven Press, 1: $55-67$

36. Malina R (1994). Physical Activity: Relationship to Growth, Maturation, and Physical Fitness. In Bouchard C, Shephard R, Stephens T (eds.). Physical Activity Fitness and Health. International Proceeding and Consensus Statement. 
Champaign: Human Kinetics Publishers, Inc., 918-930

37. Malina R (1994). Physical Activity and Training - Effects on Stature and The Adolescent Growth Spurt. Medicine and Science in Sports and Exercise 26 (6):759-766

38. Malina R, Bouchard C (1991). Growth, Maturation, and Physical Activity. Champaign: Human Kinetics Books

39. Manno R (1994) Les Qualités Physiques Entre 6 et 14 Ans. Education Physique et Sport 246: 62-65

40. Marques A, Gomes P, Oliveira J, Costa A, Graça A, Maia J (1992). Aptidão Física. In Sobral F, Marques A (eds.). FACDEX - Desenvolvimento Somato-Motor e Factores de Excelência Desportiva na População Portuguesa. Vol. 2. Relatório Parcelar Área do Grande Porto. Lisboa: Ministério da Educação - Gabinete Coordenador do Desporto Escolar, 21-43

41. Mirwald R, Bailey D, Cameron N, Rasmussen R (1981).

Longitudinal Comparison of Aerobic Power in Active and Inactive Boys Aged 7.0 to 17.0 Years. Annals of Human Biology 8 (5): 405-414

42. Parizkova J (1974). Particularities of Lean Body Mass and Fat Development in Growing Boys as Related to their Motor Activity. Acta Paediatr. Belg 28: 233-244

43. Parizkova J (1977). Body Fat and Physical Fitness. The Hague, Holland: Stenfert Kroese 198-219

44. Rico J (1994). Match Analysis. Science \& Football 8: 4-7

45. Ross W, Marfell-Jones M (1983). Kinanthropometry. In

Macdougall J, Wenger $\mathrm{H}$, Green $\mathrm{H}$ (eds.). Physiological Testing of the Elite Athlete. New York: Mouvement Publications, Inc., 75-115 46. Safrit M (1990). Introduction to Measurement in Physical Education and Exercise Science. St. Louis: Times Mirror/Mosby College Publishing

47. Schmidt R (1991). Motor Learning \& Performance - From Principles to Practice. Champaign: Human Kinetics Books 48. Sobral F (1988). O Adolescente Atleta. Lisboa: Livros Horizonte

49. Stepnicka J (1976). Somatotypes of Bohemian and Moravian Youth. Actas Facultas Medicinas Universitatis Brunensis 57: $233-242$

50. Tanner J (1962). Growth at Adolescence. Oxford: Blackwell Scientific Publication

51. Verschuur R (1987). Daily Physical Activity: Longitudinal Changes During the Teenage Period. Haarlem, Holland: Uitgeverij de Vrieseborch

52. Vrijens J, Van Cauter C (1983). Physical Performance Capacity and Specific Skills in Young Soccer Players. In Brinkhorst R, Kemper H, Saris W (eds.). International Series on Sport Sciences - Children and Exercise. XI vol.15. Champaign: Human Kinetics Publishers, Inc., 285-292

53. Zaichkowsky L, Zaichkowsky L, Martinek T (1980). Development of Motor Skills. Growth and Development. The Child and Physical Activity. London: C.V. Mosby Company, 30-55 